\title{
Aufbruch in neue Lebens-Räume: Bibel-theologische Rahmenbedingungen für die Arbeit mit ehemaligen Freiwilligen im Raum der Kirche
}

\author{
Peter Nilles \\ SOFiA e.V. | Bistum Trier $\mid$ Geschäftsführer \\ Peter.Nilles@bgv-trier.de
}

\begin{abstract}
Nicht erst seitdem die Zahl der Freiwilligen in einem internationalen Freiwilligendienst durch das weltwärts-Programm des BMZ einen deutlichen Zuwachs erfuhr, sehen Kirche und Gesellschaft in den zurückgekehrten Freiwilligen ein großes Engagementpotential, das für die weltkirchliche und entwicklungspolitische Arbeit nutzbar gemacht werden müsste. Dabei sind Kirche und Gesellschaft gleichermaßen in der Versuchung, die meist jungen Menschen für ihre jeweiligen Zwecke zu instrumentalisieren. Das Erstaunen ist dann bisweilen groß, wenn sich zeigt, dass Rückkehrer nicht so funktionieren, wie es die Akteure weltkirchlicher und entwicklungspolitischer (Bildungs-)Arbeit gerne hätten. Dieser Beitrag analysiert die Arbeit mit Rückkehrer-Freiwilligen anhand bibeltheologischer Erfahrungen und Grundvollzüge und zeigt, dass eine Orientierung an diesen Rahmenbedingungen helfen kann, dass die Arbeit mit Rückkehrerfreiwilligen besser gelingt.
\end{abstract}

Schlagwörter: Jesuanische Erfahrungen; Kirche; Rückkehrarbeit; Verzweckung von Rückkehrarbeit 


\section{Einleitung}

Wer mit zurückgekehrten Freiwilligen arbeiten will, muss zuerst wissen, wo die Freiwilligen eigentlich herkommen und was sie in ihrem Freiwilligendienst nicht nur erlebt, sondern vor allem erfahren haben. Wo kommen die Freiwilligen also her? Wer irgendwo herkommt, will auch irgendwo hin. So ist die zweite Frage, die sich jeder, der zurückgekehrten Freiwilligen begegnet, stellen sollte: Wo wollen die zurückgekehrten Freiwilligen mit dem, was sie aus dem Freiwilligendienst mitgebracht haben, hin? Das ist eine Frage, über die sich viele Rückkehrer oft selbst nicht im Klaren sind.

Wo kommen also zurückgekehrte Freiwillige her? Sie kommen aus dem Ausland, also einem Land, das außerhalb unserer inländischen Regeln und Gesetzmäßigkeiten liegt. Dort haben die Freiwilligen in der Regel vier Grunderfahrungen gemacht, die ich theologisch aus den Evangelien des Neuen Testamentes ableite. Es sind die vier jesuanischen Grunderfahrungen der Unterbrechung, der Einladung, der Berührung und der Sendung.

\section{Die vier jesuanischen Grunderfahrungen von Freiwilligen}

Die Begegnung mit einer anderen Welt ist zunächst für jeden Freiwilligen eine Unterbrechung des Gewohnten. Gewohnheiten, Regeln, Lebensweisen sind völlig anders als die, die die Freiwilligen aus Deutschland kennen. Unterbrechung ist eine zutiefst biblische Erfahrung. Das Auftreten Jesu ist eine Unterbrechung. Seine Botschaft ist eine Unterbrechung. Wer Gefangene in Freiheit setzen will - so in seiner programmatischen Rede in der Synagoge von Nazareth (Lk 4, 18-19) ${ }^{1}$, stört die Kreise der Mächtigen. Von dem katholischen Theologen Johann Baptist Metz stammt der Ausspruch, dass Unterbrechung die kürzeste Definition von Religion ist (Noormann/Becker/Trocholepczy 2007: 19). Die extremste Form der Unter-

\footnotetext{
${ }^{1}$ Unter Rückgriff auf den Propheten Jesaja formuliert Jesus im Lukasevangelium sein Programm folgendermaßen: Der Geist des Herrn ruht auf mir; / denn der Herr hat mich gesalbt. Er hat mich gesandt, / damit ich den Armen eine gute Nachricht bringe; damit ich den Gefangenen die Entlassung verkünde / und den Blinden das Augenlicht; damit ich die Zerschlagenen in Freiheit setze und ein Gnadenjahr des Herrn ausrufe.
} 
brechung ist der Tod. Noch vor jedem öffentlichen Auftreten Jesu steht die Erfahrung der Unterbrechung; Jesus geht 40 Tage in die Wüste.

Die Freiwilligen machen vielfach die Erfahrung der Unterbrechung und Störung in ihrem Freiwilligendienst. Für die erste Unterbrechung entscheiden sie sich noch selbst, wenn sie ins Ausland gehen. Alle anderen Unterbrechungen kommen auf sie $\mathrm{zu}$, ob das sprachliche und gesundheitliche Probleme sind oder ob es die zumutende Begegnung mit Menschen in Elend und Armut ist. Sie machen auch Wüstenerfahrungen, wenn sie oft auf sich allein angewiesen sind. Jede Unterbrechung ist zunächst einmal störend, weil die Freiwilligen sich diese nicht aktiv ausgesucht haben. Jede Erfahrung kommt auf sie zu; das ist der Charakter von Erfahrungen.

Erfahrungen kann man annehmen oder ablehnen. Wer sich als Freiwilliger auf die Erfahrung der Unterbrechung einlässt, hat damit die Chance, die zweite Erfahrung der Einladung zu machen. Auch dies ist eine zutiefst jesuanische Erfahrung. Im Neuen Testament wird Jesus immer wieder zu Gastmählern mit den Sündern, den Randexistenzen der damaligen Gesellschaft eingeladen. Die Typen, die zunächst stören - Zöllner, Dirnen - Leute also, die das Gewohnte unterbrechen und damit stören, erweisen sich bei näherem Hinsehen als Menschen, von denen und deren Lebensgeschichte eine tiefe Einladung ausgeht.

Das ist die Erfahrung, die auch Freiwillige im Freiwilligendienst machen, wenn sie sich denn auf die Erfahrung der Unterbrechung einlassen. Sie begegnen im Ausland Menschen, von denen und deren Lebensgeschichte eine Einladung und Faszination ausgeht. Dabei spüren sie, dass diese ihnen mehr schenken, als sie ihnen ihrerseits geben können. Auch die Erfahrung der Einladung kann man annehmen oder ausschlagen. Wer sich als Freiwilliger darauf einlässt, hat die Chance, wiederum eine dritte Erfahrung zu machen. Das ist die Erfahrung der Berührung.

Im Neuen Testament ist von Berührung immer im Zusammenhang mit den Heilungsgeschichten die Rede. Jesus wird in der Regel nicht aufgefordert, kranke Menschen zu heilen, sondern sie zu berühren. Von seiner Berührung geht eine heilende Kraft aus. Zum Teil handelt es sich dabei um sehr elementare Berührungen, etwa wenn Jesus die Zunge des Taubstummen mit seiner eigenen Spucke berührt oder wenn er mit der gleichen Spucke im 
Staub der Erde einen Schlamm anrührt, mit dem er die Augen des Blinden bestreicht.

Die Freiwilligen im Auslandsdienst machen ähnlich elementare Berührungserfahrungen. Sie kommen in Berührung - mit Schweiß und Staub, mit Gerüchen und Anblicken von Menschen und Lebenswirklichkeiten, die ihnen elementare Berührungen zumuten. Von Menschen handgreiflich angefasst zu werden, umarmt zu werden, berührt nicht nur die Haut, sondern geht unter die Haut und berührt die Herzen der Freiwilligen.

So wie die Erfahrung der Unterbrechung und der Einladung kann man auch die Erfahrung der Berührung entweder annehmen oder ablehnen. Wer sich von den Menschen und ihrem Leben am Herzen berühren lässt, hat schließlich die Chance, die vierte jesuanische Erfahrung der Sendung zu machen.

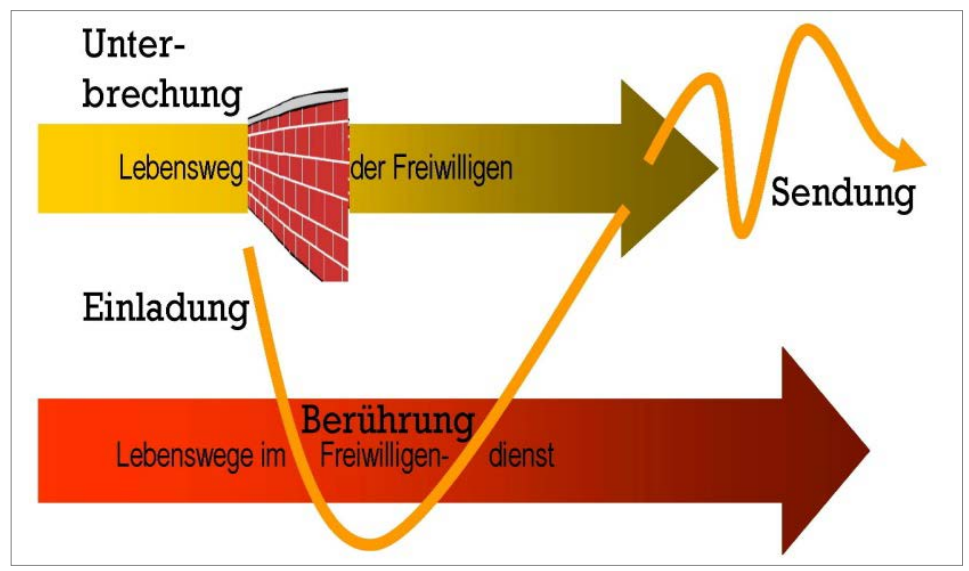

Abb. 1: Die vier jesuanischen Grunderfahrungen von Freiwilligen;

Quelle: Eigene Darstellung

Das ist die Erfahrung der Emmaus-Jünger, die - nachdem Jesus sich unterwegs mit ihnen unterhalten hatte und plötzlich nicht mehr da ist - feststellen: Brannte nicht das Herz in unserer Brust, als er unterwegs mit uns redete. Aus der Retrospektive bekommen ihre Erfahrungen mit Jesus Deutung, Sinn und eine Richtung. Nichts ist wie vorher. Genau diese Erfahrung machen Freiwillige, wenn sie nach der Rückkehr aus dem Ausland nach Deutschland zurückkehren. In ihrem Herzen brennt es. Nichts ist wie vor ihrer Ausreise ins Ausland. In den vielfach unterbrechenden und berührenden Erfahrungen 
haben sie eine paradoxe Fülle an beglückendem Leben entdeckt, die sie vorher nicht kannten und nicht einmal erahnt hätten.

All dieses muss man wissen, wenn man mit Freiwilligen nach ihrer Rückkehr in Deutschland arbeiten will.

Freiwillige kommen quasi quer von der Seite aus einer völlig anderen Welt in eine geordnet und geradlinig verlaufende und strukturierte Lebenswelt, in der sie mit ihren Erfahrungen überall anecken und kollidieren. Sie stehen vor der großen Herausforderung, das, was für sie wichtig geworden ist, in den

Grenzen der ,alten“ deutschen Gesellschaft leben zu können und noch öfter leben zu müssen. Dabei brechen sie mit ihrer Dynamik immer wieder aus den Grenzen der neuen alten Gesellschaft aus.

Auf diesem Hintergrund versteht es sich von selbst, dass Rückkehrer in dieser Situation keinesfalls im Sinne einer zielorientierten weltkirchlichen und/oder entwicklungspolitischen (Bildungs-)Arbeit funktionieren können. Sie passen nicht ins System und stellen vieles in Frage. Was ist also zu beachten, damit die Arbeit mit Rückkehrern gelingen kann? Auch hier kann das Neue Testament Orientierung geben.

\section{Die kirchlichen Grundvollzüge als Rahmen für die Arbeit mit Rückkehrfreiwilligen}

Im Prinzip haben die Jünger mit Jesus über drei Jahre nichts anderes als einen Freiwilligendienst absolviert. Sie haben die Erfahrungen der Unterbrechung, der Einladung und Berührung mit ihm gemacht und sind in Emmaus mit der Erfahrung der Sendung konfrontiert. Spätestens bei der Himmelfahrt Jesu wurden sie in ihre Heimatdörfer in Galiläa zurückgeschickt.

Die Geschichte endet jedoch nicht am Ende des Evangeliums in Emmaus. Ganz im Gegenteil beginnt mit der Apostelgeschichte die Zeit, in der sie sich zunächst zurückziehen, aber dann ihre Erfahrungen nicht für sich behalten, sondern mit anderen Menschen teilen. Das ist theologisch die Zeit der Kirche, die sich durch vier ineinandergreifende kirchliche Grundvollzüge auszeichnet. Es sind die Grundvollzüge der koinonia, martyria, diakonia und leiturgia, die auch den Rahmen für jegliche Arbeit mit Rückkehrerfreiwilligen abstecken. 
Koinonia meint die Gemeinschaft der Gleichgesinnten, in der man Verständnis, Austausch, Vergewisserung und Identifikation sucht. Koinonia ist der Ort, an dem die gemachten Erfahrungen artikuliert, gedeutet und in das eigene Leben integriert werden können. Das geschieht nicht immer harmonisch, bisweilen kontrovers und diskursiv.

Zurückgekehrte Freiwillige suchen genau diese Räume. Sie suchen den Kontakt und Austausch mit Menschen, die die gleichen Erfahrungen wie sie gemacht haben. Sie suchen Menschen, die sie und ihre Lebenssituation verstehen und die ihnen Hilfestellung geben, sie selbst zu verstehen. In ihrer ihnen oft fremd gewordenen privaten Umgebung stoßen sie vielfach auf Unverständnis und Desinteresse. Koinonia ist für Rückkehrerfreiwillige ein ganz wichtiger Grundvollzug des Lebens.

Der zweite Grundvollzug von Kirche ist die martyria, das Zeugnis ablegen. Es ist das Bedürfnis, das was einem wichtig geworden ist, zu vergewissern und mit anderen zu teilen, mit-zu-teilen. Die Jünger wollen das, was sie mit Jesus erlebt haben, keinesfalls nur für sich behalten. Sie sind davon so überzeugt, dass sie es auch gegen die Mehrheitsmeinung vertreten.

Auch das ist ein Grundvollzug von Rückkehrerfreiwilligen. In ihrem Freiwilligendienst sind ihnen Menschen und Lebensvollzüge selbstverständlich und wichtig geworden. Sie haben Menschen kennen gelernt, die ihnen ans Herz gewachsen sind - ob das Menschen in Syrien sind, die unter Krieg leiden oder Menschen in Nigeria, die bereits den Weg durch die Sahara gegangen sind, um von Nordafrika mit dem Boot nach Europa zu gelangen. Eine in Westeuropa vielfach verbreitete Indifferenz ist Standpunkten gewichen, die Freiwillige für ihnen vertraut gewordene Menschen in anderen Ländern beziehen. Diese Standpunkte sind gesellschaftlich oft nicht opportun, sondern stehen vielfach gegen den Mainstream. Rückkehrerfreiwillige beziehen vielfach nonkonform Position. Martyria ist ebenfalls ein elementarer Grundvollzug im Leben von Rückkehrerfreiwilligen.

Der dritte Grundvollzug von Kirche ist die diakonia, das soziale Engagement für die Schwachen. Es reicht nicht aus, Position zu beziehen. Handeln ist gefragt. Rückkehrerfreiwillige haben vielfach das Bedürfnis, sich weiter für die Menschen in ihren Einsatzländern zu engagieren. Sie schließen sich zusammen, gründen Vereine, schließen sich Initiativen an, initiieren selbst Unterstützungs- und Hilfsmaßnahmen und informieren über ungerechte 
Verhältnisse. Diakonia, also sich zu engagieren ist für Freiwillige ein Bedürfnis und letztlich Grundvollzug ihres Lebens.

Die drei Grundvollzüge, koinonia, martyria und diakonia, fließen schließlich zusammen in der leiturgia, dem vierten kirchlichen Grundvollzug der (gottesdienstlichen) Feier. Diese Art zu leben will gefeiert, also verdichtet und ausgedrückt sein. So ist Feier nicht nur der Zusammenfluss der Grundvollzüge kirchlichen Lebens, sondern gleichzeitig auch Ausgangspunkt für die Fortsetzung des Lebens, gleichsam Ausatmen wie Einatmen.

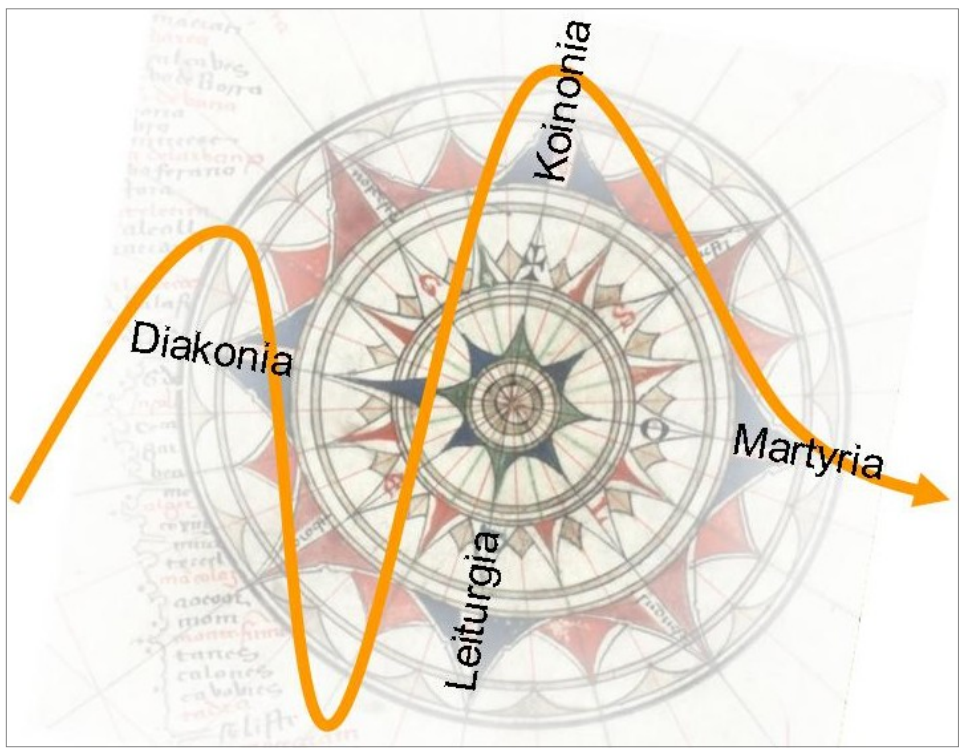

Abb. 2: Die Grundvollzüge der Kirche als Rahmen für die Arbeit mit RückkererFreiwilligen; Quelle: Eigene Darstellung

Nichts tun Rückkehrerfreiwillige lieber als das Leben zu feiern. Immer wieder suchen sie Orte und Gelegenheiten, wo sich ihre Art zu leben verdichtet und damit neue Impulse erhält. Feier, also leiturgia ist auch für Freiwillige ein wesentlicher Grundvollzug ihres Lebens.

Die vier genannten Grundvollzüge greifen in der Arbeit mit Rückkehrern ineinander. Sie müssen ineinander greifen, weil sie sich gegenseitig bedingen. Eine reine Reduktion der Rückehrerarbeit auf die Feier (leiturgia) und Gemeinschaft (koinonia) verliert ihren Sinn, wenn es nichts zu feiern gibt. 
Wenn man sich dabei weder des eigenen Standpunktes vergewissern kann (martyria) noch Kraft für das Engagement für andere schöpft (diakonia).

Eine reine Reduktion der Rückkehrerarbeit auf Standpunkt und Position beziehen bleibt für die Freiwilligen unbefriedigend und zehrt aus, wenn nicht komplementär Möglichkeiten bestehen, sich zu engagieren (diakonia) bzw. die eigenen Positionen zu vergewissern (koinonia) oder in der Feier zu verdichten (leiturgia).

Eine reine Reduktion der Rückkehrerarbeit auf den Aktionismus sozialen Engagements verliert Sinn und Richtung, wenn es keine Gelegenheit gibt, den eigenen Standpunkt zu artikulieren (martyria) bzw. sich mit anderen immer wieder zu vergewissern, ob man mit seinem Engagement noch auf dem richtigen Weg ist (koinonia, leiturgia).

Nur dort, wo alle vier kirchlichen Grundvollzüge in der Rückkehrerarbeit ineinander greifen und sich gegenseitig befruchten und ergänzen, kann Rückkehrerarbeit gelingen und wird Rückkehrerarbeit zum lebendigen Ort vielleicht auch zum Modell - gelungenen Kirche-seins. Auf diesem Hintergrund verbietet es sich von selbst, Freiwillige lediglich für bestimmte, auch noch so gut gemeinte Zwecke abzurufen, ohne ihnen die Lebens- und Entfaltungsräume, die sie bei der Suche nach ihrer Sendung (Berufung) brauchen, anzubieten und zu öffnen. Vielmehr sollten sich die verschiedenen kirchlichen Akteure bei der Gestaltung der Rückkehrerarbeit so ergänzen, dass der Rahmen für die Lebensvollzüge von Rückkehren in ausreichendem Maße gewährleistet ist

\section{Literaturverzeichnis}

Noormann, Harry, Ulrich Becker und Bernd Trocholepczy (Hrsg.) (2007): Ökumenisches Arbeitsbuch Religionspädagogik, 3. Aufl., Stuttgart. 
Voluntaris, Jg. 1, 1/2013, Aufsätze

\begin{abstract}
Peter Nilles

Departure into new spheres of life: Work with former volunteers in the sphere of the Church

Not only since the number of volunteers in an international volunteers service experienced a clear growth through the weltwärts ("worldwards") programme launched by the Federal Ministry for Economic Cooperation and Development (BMZ) Church and society have seen a great potential of commitment in returned volunteers which should be transformed in practical use for universal church and development aid policy work. Here Church and society are equally tempted to instrumentalise the mostly young people for their respective purposes. The astonishment is then from time to time great when it turns out that the returned volunteers do not function quite the way the protagonists of universal church and development aid policy (educational) work would like them to. This article analyses the work with returned volunteers on the basis of bible theological experiences and basic principals, and shows that an orientation towards these prevailing conditions can help to work with returned volunteers to succeed better.
\end{abstract}

Keywords: Returnee Work, Jesuanic Experiences, Bible, Church, Profile, Spirituality 